\author{
Hanno Hoppe \\ Sara Spagnuolo \\ Johannes M. Froehlich \\ Helga Nievergelt \\ Hans-Peter Dinkel \\ Silvia Gretener \\ Harriet C. Thoeny
}

\section{Retrospective analysis of patients for development of nephrogenic systemic fibrosis following conventional angiography using gadolinium-based contrast agents}

Received: 22 April 2009

Revised: 30 June 2009

Accepted: 6 August 2009

Published online: 16 September 2009

(C) European Society of Radiology 2009

H. Hoppe $(\bowtie) \cdot$ S. Spagnuolo ·

J. M. Froehlich · H. C. Thoeny

Institute of Diagnostic, Interventional, and Pediatric Radiology, Inselspital,

University Hospital Bern,

Freiburgstrasse 10,

3010 Bern, Switzerland

e-mail: hanno.hoppe@insel.ch

Tel.: +41-31-6322435

Fax: +41-31-6324874

\section{H. Nievergelt}

Clinic of Dermatology,

University Hospital Bern,

Bern, Switzerland

\section{H.-P. Dinkel}

Institute of Diagnostic and Interventional Radiology,

Hospital Landshut,

Landshut, Germany

\section{S. Gretener}

Division of Vascular Medicine,

Swiss Cardiovascular Center,

University Hospital of Bern,

Bern, Switzerland
Abstract Purpose: The purpose was to retrospectively review the data of 27 patients with renal insufficiency who underwent conventional angiography with gadolinium-based contrast agents (GDBCA) as alternative contrast agents and assess the occurrence of nephrogenic systemic fibrosis (NSF) together with associated potential risk factors. Methods: This HIPAA-compliant study had institutional review board approval, and informed consent was waived. Statistical analysis was performed for all available laboratory and clinical data, including dermatology reports. Type and amount of the GDBCA used were recorded for angiography and additional MRI studies, if applicable.

Serum creatinine levels ( $\mathrm{SCr}$ ) pre- and post-angiography were recorded, and estimated glomerular filtration rates (eGFR) were calculated. Results: Ten female and 17 male patients who underwent angiography with GDBCA were included. The mean amount of GDBCA administered was $44 \pm$
$15.5 \mathrm{ml}$ (range $15-60 \mathrm{ml}$ ) or $0.24+$ $0.12 \mathrm{mmol} / \mathrm{kg}$ (range $0.1-0.53 \mathrm{mmol} /$ $\mathrm{kg}$ ). At the time of angiography all patients had renal insufficiency (eGFR $\left.<60 \mathrm{ml} / \mathrm{min} / 1.73 \mathrm{~m}^{2}\right)$. Mean eGFR pre-angiography was $26 \mathrm{ml} /$ $\min / 1.73 \mathrm{~m}^{2}$ and $33 \mathrm{ml} / \mathrm{min} / 1.73 \mathrm{~m}^{2}$ post-angiography. The mean followup period covers 28 months, range 184 months. Additional MRI studies with GDBCA administration were performed in 15 patients. One patient with typical skin lesions had developed biopsy-confirmed NSF.

Conclusion: Conventional arterial angiography with GDBCA may play a role in the development of NSF in patients with renal insufficiency. Alternative contrast agents, such as $\mathrm{CO}_{2}$ angiography or rather the use of low doses of iodinated contrast agents, should be considered in these patients.

Keywords Contrast media . Angiography $\cdot \mathrm{NSF} \cdot$ Gadolinium · Renal insufficiency

\section{Introduction}

It is well known that the use of iodinated contrast material may induce nephropathy, especially in patients with underlying renal insufficiency [1]. In the past, gadolinium-based contrast agents (GDBCA) were used as alternative contrast agents for conventional angiography in patients with renal insufficiency on an off-label basis, although the
Contrast Media Safety Committee (CMSC) of the European Society of Urogenital Radiology (ESUR) did not approve GDBCA for radiographic examinations $[2,3]$. In a previous study, it was concluded that angiography and percutaneous treatment of renal artery stenosis in patients with chronic renal insufficiency and suspected ischaemic nephropathy can be performed safely using intra-arterial injections of GDBCA as contrast 
media without an increased risk of complications. Contrast-induced nephropathy potentially occurred in no more than $3.2 \%$ of patients [4]. It was further noted that neither the degree of underlying renal insufficiency nor diabetes was a risk factor for predicting a greater likelihood of renal function impairment at 48-h followup [4].

Recently, an illness called nephrogenic systemic fibrosis (NSF) or nephrogenic fibrosing dermopathy was described in patients with renal impairment presenting with typical skin lesions and joint disease [5]. Fulminate progression with systemic complications and autopsy reports even suggested that NSF is a systemic disorder that may involve other organs resulting in severe damage or even death $[6,7]$. Of interest, a strong relationship between administration of GDBCA and an outbreak of NSF was suggested by various reports in the literature [8-10]. Even though it has not been proven that gadolinium is the triggering agent, gadolinium administration could be established in most NSF cases.

The purpose of this study was to retrospectively review clinical and laboratory data of 27 patients with renal insufficiency who underwent conventional angiography with GDBCA as alternate contrast media and assess for the occurrence of NSF.

\section{Materials and methods}

\section{Patients}

This HIPPA-compliant study was approved by our institutional review board, and informed consent was waived. As of February 2007, we retrospectively analysed data of 27 consecutive patients (10 women and 17 men, median age 77 years, age range 20-97 years) who had received GDBCA as an alternative contrast agent for conventional angiography between June 2000 and January 2002 on an off-label basis. Of those, 17 patients also received $\mathrm{CO}_{2}$ and 13 patients also received iodinated contrast material, including 8 patients who received both $\mathrm{CO}_{2}$ and iodinated contrast material. All patients were preand post-hydrated intravenously with 11 of saline solution $(0.9 \%)$ each time.

\section{Data collection}

In the 27 patients, all available clinical and laboratory data were collected. Patient's primary care physicians were called to collect the most recent patient information. Data collected included age, gender, type and date of angiography and intervention, type and amount of contrast material administered, additional MRI studies with contrast administration, signs of NSF noted on the chart including dermatology reports and biopsy results, relevant comorbidities, cause and stage of kidney disease, and dialysis/kidney transplant status. Skin biopsies were stained with haematoxylin and eosin and immunohistochemistry with CD 34 and CD 68 antibodies, both characteristic of NSF. The laboratory values examined included serum creatinine ( $\mathrm{sCr}$ ) levels pre- and postangiography, and estimated glomerular filtration rates (eGFR). Based on the National Kidney Foundation Kidney Disease Outcomes Quality Initiative, kidney disease was staged as follows: stage 1: eGFR $>90 \mathrm{ml} / \mathrm{min} / 1.73 \mathrm{~m}^{2}$, stage 2: eGFR $60-90 \mathrm{ml} / \mathrm{min} / 1.73 \mathrm{~m}^{2}$, stage $3: 30-59 \mathrm{ml} /$ $\mathrm{min} / 1.73 \mathrm{~m}^{2}$, stage $4: 15-29 \mathrm{ml} / \mathrm{min} / 1.73 \mathrm{~m}^{2}$ and stage 5: $<15 \mathrm{ml} / \mathrm{min} / 1.73 \mathrm{~m}^{2}$.

\section{Statistical analysis}

The age and gender distribution of patients was calculated using descriptive statistics. Mean, standard deviation and range were calculated for the amount of gadolinium administered during angiography, serum creatinine values and eGFR. Analysis was performed with GraphPad statistical software (Prism v. 4.01 and InStat v 3.0; GraphPad Software, San Diego, CA).

\section{Results}

Results are summarised in Tables 1 and 2. Twenty-five patients received low-osmolar linear chelate gadodiamide (Omniscan, GE Healthcare, New York, NY). The mean amount of gadodiamide administered intra-arterially was $44 \pm 15.5 \mathrm{ml}$ (range $15-60 \mathrm{ml}$ ) or $0.24+0.12 \mathrm{mmol} / \mathrm{kg}$ (range $0.1-0.53 \mathrm{mmol} / \mathrm{kg}$ ). One patient received $35 \mathrm{ml}$ $(0.23 \mathrm{mmol} / \mathrm{kg})$ of gadopentetate (gadolinium-DTPA; Magnevist, Bayer Schering AG, Leverkusen, Germany) and another patient received $20 \mathrm{ml}(0.3 \mathrm{mmol} / \mathrm{kg})$ of gadobutrol (Gadovist $1.0 \mathrm{mmol} / \mathrm{ml}$, Bayer Schering AG, Leverkusen, Germany). Seventeen patients also received $\mathrm{CO}_{2}$ besides GDBCA, and 13 patients were co-administered with relatively small amounts of iodinated contrast material (mean $14.3 \pm 16.7 \mathrm{ml}$, range $5-60 \mathrm{ml}$ ), including 8 patients who received both $\mathrm{CO}_{2}$ and iodinated contrast material. Additional MRI studies with GDBCA administration were performed in 15 patients (Table 1).

At the time of angiography all patients had at least stage 3 renal insufficiency (eGFR $<60 \mathrm{ml} / \mathrm{min} / 1.73 \mathrm{~m}^{2}$ ). The mean eGFR pre-angiography was $26.2 \pm 13.1 \mathrm{ml} /$ $\mathrm{min} / 1.73 \mathrm{~m}^{2}$ (Table 2). According to the National Kidney Foundation Kidney Disease Outcome Quality Initiative, 8 patients were stage 3, 13 stage 4 , and 6 stage 5 . The median stage of disease was 4 (range 3-5). The mean ultimate eGFR post-angiography (mean 28 months) was $33 \pm$ $18.6 \mathrm{ml} / \mathrm{min} / 1.73 \mathrm{~m}^{2}$. Mean $\mathrm{sCr}$ levels were $263.1 \pm$ $128.7 \mu \mathrm{mol} / \mathrm{l}$ (range 134-728 $\mu \mathrm{mol} / \mathrm{l}$ ) pre-angiography, $288.2 \pm 154.7 \mu \mathrm{mol} / 1$ (range $125-764 \mu \mathrm{mol} / \mathrm{l}) 2 \mathrm{~h}$ post angiography, $261.7 \pm 156.6 \mu \mathrm{mol} / \mathrm{l}$ (range $127-712 \mu \mathrm{mol} / \mathrm{l}$ ) 
Table 1 Demographic and clinical data in 27 patients post-digital subtraction angiography with gadolinium-based contrast agents (GDBCA)

\begin{tabular}{|c|c|c|c|c|c|}
\hline No./sex & $\begin{array}{l}\text { Angiography } \\
\text { with GDBCA }\end{array}$ & $\begin{array}{l}\text { Dose }(\mathrm{ml}) / \text { type } \\
\text { of GDBCA }\end{array}$ & $\begin{array}{l}\text { Additional MRI } \\
\text { with GDBCA }\end{array}$ & $\begin{array}{l}\text { Dose }(\mathrm{ml}) / \text { type } \\
\text { of GDBCA }\end{array}$ & $\begin{array}{l}\text { Concurrent medical conditions at } \\
\text { time of angiography with GDBCA }\end{array}$ \\
\hline $1 / \mathrm{F}$ & DSA and PTA lower extremity & 35 Magnevist & N/A & N/A & DM, AOD \\
\hline $2 / \mathrm{M}$ & DSA lower extremity & 50 Omniscan & $\begin{array}{l}\text { Abdomen } \\
\text { Head }\end{array}$ & $\begin{array}{l}50 \text { Omniscan } \\
20 \text { Magnevist }\end{array}$ & AOD, CP, SCLC, DM, cvi \\
\hline $3 / \mathrm{M}$ & DSA lower extremity & 20 Omniscan & N/A & N/A & AOD, PPN, CU, CCP, COPD, GERD, BR \\
\hline $4 / F$ & DSA and PTA lower extremity & 36 Omniscan & N/A & N/A & AOD, sA, DM, CCP, COPD \\
\hline $5 / F$ & DSA and PTA lower extremity & 36 Omniscan & N/A & N/A & AOD, aHT, AS, HP, OP, SCS, NNA \\
\hline $6 / F$ & DSA and PTA lower extremity & 20 Omniscan & Renal & 20 ProHance & $\mathrm{AOD}, \mathrm{DM} 2, \mathrm{CCP}, \mathrm{cP}, \mathrm{A}$ \\
\hline $7 / \mathrm{M}$ & DSA and PTA lower extremity & 20 Omniscan & $\begin{array}{l}\text { Renal } \\
\text { Abdomen }\end{array}$ & $\begin{array}{l}15 \text { ProHance } \\
30 \text { Omniscan }\end{array}$ & AOD, NI, CCP, IDP \\
\hline $8 / \mathrm{F}$ & DSA abdomen & 30 Omniscan & Renal & 25 Omniscan & CS, cNI, CCP, DM, CVI, A \\
\hline \multirow[t]{2}{*}{ 9/M } & $\begin{array}{l}\text { DSA and PTA pelvis and } \\
\text { lower extremity }\end{array}$ & 20 Omniscan & Pelvis & 20 ProHance & ASCL, AOD, DM, COPD, NSCLC \\
\hline & & & $\begin{array}{l}\text { Pelvis } \\
\text { Renal } \\
\text { Renal } \\
\text { Hip joint } \\
\text { Abdomen }\end{array}$ & $\begin{array}{l}20 \text { Dotarem } \\
30 \text { Omniscan } \\
30 \text { Omniscan } \\
20 \text { Dotarem } \\
50 \text { Omniscan }\end{array}$ & \\
\hline \multirow[t]{2}{*}{$10 / \mathrm{M}$} & $\begin{array}{l}\text { DSA and PTRA renal } \\
\text { transplant }\end{array}$ & 15 Omniscan & Head & 15 Magnevist & aHT, DM2, hCP, hc, Ac \\
\hline & & & $\begin{array}{l}\text { Abdomen } \\
\text { Renal } \\
\text { Abdomen } \\
\text { Abdomen } \\
\text { Abdomen } \\
\text { Neck } \\
\text { Renal } \\
\text { Renal }\end{array}$ & $\begin{array}{l}20 \text { ProHance } \\
10 \text { MultiHance } \\
7 \text { MultiHance } \\
20 \text { ProHance } \\
30 \text { Omniscan } \\
15 \text { Gadovist } \\
30 \text { Omniscan } \\
30 \text { Omniscan }\end{array}$ & \\
\hline $11 / \mathrm{M}^{*}$ & DSA renal transplant & 15 Omniscan & $\begin{array}{l}\text { Renal } \\
\text { Renal } \\
\text { Femur } \\
\text { Renal } \\
\text { Renal } \\
\text { Renal } \\
\text { Renal } \\
\text { Knee joint }\end{array}$ & $\begin{array}{l}30 \text { ProHance } \\
30 \text { Omniscan } \\
30 \text { ProHance } \\
30 \text { Omniscan } \\
30 \text { Omniscan } \\
30 \text { Omniscan } \\
20 \text { ProHance } \\
10 \text { Magnevist }\end{array}$ & hsm, ta, crf, aHT, HP, rA \\
\hline $12 / \mathrm{M}$ & DSA pelvis and lower extremity & 20 Omniscan & Neck & 15 Gadovist & AOD, CCP, cNI, Hy \\
\hline $13 / \mathrm{F}$ & $\begin{array}{l}\text { DSA and PTA pelvis and } \\
\text { lower extremity }\end{array}$ & 30 Omniscan & $\mathrm{N} / \mathrm{A}$ & N/A & AOD, MM, CCP, NNA, UC \\
\hline $14 / \mathrm{M}$ & $\begin{array}{l}\text { DSA and PTRA abdomen } \\
\text { and kidney }\end{array}$ & 40 Omniscan & Renal & 30 Omniscan & CoxA, HU, dCP, GITB, LR \\
\hline $15 / \mathrm{M}$ & DSA lower extremity & 20 Omniscan & Renal & 30 Omniscan & CoSy, aHT, MI, PCA, TA, GIB, AAA, Tbc \\
\hline \multirow[t]{3}{*}{$16 / F$} & $\begin{array}{l}\text { DSA and PTRA abdomen } \\
\text { and kidney }\end{array}$ & 30 Omniscan & Renal & 30 ProHance & aHT, rA, HP \\
\hline & & & Abdomen & 30 ProHance & \\
\hline & & & Abdomen & 15 ProHance & \\
\hline
\end{tabular}




\begin{tabular}{|c|c|c|c|c|c|}
\hline No./sex & $\begin{array}{l}\text { Angiography } \\
\text { with GDBCA }\end{array}$ & $\begin{array}{l}\text { Dose }(\mathrm{ml}) / \text { type } \\
\text { of GDBCA }\end{array}$ & $\begin{array}{l}\text { Additional MRI } \\
\text { with GDBCA }\end{array}$ & $\begin{array}{l}\text { Dose }(\mathrm{ml}) / \text { type } \\
\text { of GDBCA }\end{array}$ & $\begin{array}{l}\text { Concurrent medical conditions at } \\
\text { time of angiography with GDBCA }\end{array}$ \\
\hline \multirow[t]{2}{*}{$17 / \mathrm{M}$} & $\begin{array}{l}\text { DSA and PTRA abdomen } \\
\text { and kidney }\end{array}$ & 20 Omniscan & Head & 20 Magnevist & RAS, CCP, ATH, HP, aHT, REA \\
\hline & & & Renal & 30 ProHance & \\
\hline \multirow[t]{2}{*}{$18 / \mathrm{M}$} & DSA and PTA pelvis & 20 Gadovist & Renal & 30 Omniscan & rA, aHT, HC, ATN, NNA, LC \\
\hline & & & Head & 15 Magnevist & \\
\hline $19 / \mathrm{F}$ & DSA and PTA lower extremity & 40 Omniscan & N/A & N/A & N/A \\
\hline $20 / \mathrm{M}$ & $\begin{array}{l}\text { DSA and PTA abdomen } \\
\text { and lower extremity }\end{array}$ & 90 Omniscan & N/A & N/A & AOD, aHT, NI, LHI, CP, COPD \\
\hline \multirow[t]{3}{*}{$21 / \mathrm{F}$} & DSA and PTA lower extremity & 40 Omniscan & Foot & 8 Omniscan & AOD, MNHL, HMA, Cni, D, Hy, cO, GA \\
\hline & & & Ankle joint & 9 ProHance & \\
\hline & & & Pelvis & 30 Omniscan & \\
\hline $22 / \mathrm{M}$ & DSA abdomen & 60 Omniscan & N/A & $\mathrm{N} / \mathrm{A}$ & crf, CCP, MI, pHT, AAA \\
\hline $23 / \mathrm{F}$ & DSA and PTA lower extremity & 20 Omniscan & N/A & N/A & UC, DM2, dCP, PE, DVT, CVI \\
\hline $24 / \mathrm{M}$ & DSA lower extremity & 35 Omniscan & N/A & N/A & CCP, MI, DM, Trf, EP \\
\hline $25 / \mathrm{M}$ & DSA lower extremity & 30 Omniscan & N/A & N/A & DM, aHT, RCA \\
\hline $26 / \mathrm{M}$ & $\begin{array}{l}\text { DSA and PTRA abdomen } \\
\text { and kidney }\end{array}$ & 50 Omniscan & Abdomen & 30 Magnevist & CCP, AP, COPD, aHT, AOD, TIA, TURP \\
\hline $27 / \mathrm{M}$ & DSA abdomen and pelvis & 60 Omniscan & N/A & N/A & ASKL, AOD \\
\hline
\end{tabular}

*This patient demonstrated NSF-typical skin changes that were biopsy-proven

Abbreviations:

A, adiposity; AAA, abdominal aortic aneurysm; Ac, acne; aHT, arterial hypertension; AOD, arterial occlusive disease; AP, angina pectoris; AS, aortic stenosis; ASCL, arteriosclerosis; ATN, acute tubular necrosis; BR, Billroth's stomach; CCP, coronary cardiopathy; COPD, chronic obstructive pulmonary disease; CoSy, compartment syndrome $\mathrm{M}$. triceps surae; CoxA, coxarthritis; $\mathrm{CP}$, cardiopathy; $\mathrm{cP}$, chronic pancreatitis; crf, chronic renal failure; CS, carotid stenosis; CU, Cushing's disease; cvi, cerebrovascular insult; CVI, chronic venous insufficiency; dCP, dilatative cardiomyopathy; DM, diabetes mellitus; DVT, deep vein thrombosis; EP, encephalopathy; F, female; GDBCA, gadolinium-based contrast agent; GERD, gastro-oesophageal reflux disease; GIB, gastrointestinal bleeding; HC, haemochromatosis; hc, hypercholesterinaemia; HMA, hyperchromic macrocytic anaemia; HP, hyperparathyroidism; hsm, hepatosplenomegaly; HU, hyperuricaemia; Hy, hypothyreosis; IDP, intervertebral disk prolapse; LC, lymphocele; LHF, left heart failure; LR, livedo racemosa; M, male; MI, myocardial infarction; MM, multiple myeloma; MNHL, malignant non-Hodgkin's lymphoma; N/A, not applicable; NNA, normochromic normocytic anaemia; NSCLC, non-small cell lung cancer; OP, osteoporosis; PCA, prostatic cancer; PE, pulmonary embolus; pHT, pulmonary hypertension; PPN, peripheral polyneuropathy; rA, renal anaemia; RAS, renal artery stenosis; RCA, rectal cancer; REA, reanimation, sA, septic arthritis; SCLC, small cell lung cancer; SCS, spinal canal stenosis; ta, tachyarrhythmia; TA, temporal arteritis; Tbc, tuberculosis; TIA, transient ischaemic attack; Trf, terminal renal failure; TURP, transurethral resection of the prostate; UC, ulcera cruris

2 weeks post angiography, $310.3 \pm 179.5 \mu \mathrm{mol} / 1$ (range $122-592 \mu \mathrm{mol} / 1) 3$ months post-angiography and final $\mathrm{sCr}$ level after angiography was $247.1 \pm 181.8 \mu \mathrm{mol} / \mathrm{l}$ (range $78-880 \mu \mathrm{mol} / \mathrm{l})$. The mean follow-up period was 28 $( \pm 29.5)$ months, range 1-84 months.

Dermatology reports were available for 11 patients. Only one patient chart contained evidence of NSF. This 20 -year old patient (no. 11 in Tables 1 and 2, patient data summary in Table 3) was on dialysis from October 1998 to August 2000. He received a renal transplant in August 2000 , and dialysis was stopped. This patient had multiple studies with GDBCA post-renal transplantation receiving both linear and macrocyclic gadolinium-based contrast agents (Table 3). The patient had to go back on dialysis in May 2001. He observed skin changes and developed joint pain in his knees, elbows and hands in September 2001, which was approximately 5 months post-renal transplant angiography with GDBCA. At the time, this patient had received a total of $215 \mathrm{ml}$ of GDBCA, including $135 \mathrm{ml}$ of linear and $80 \mathrm{ml}$ of macrocyclic contrast agents. A skin biopsy taken in late December 2001 was positive for NSF in retrospect. Workup of this patient's joint pain included additional MRI studies of his thigh and knees with GDBCA demonstrating synovial proliferation and periosteal inflammation. Macroscopic skin examination demonstrated generally dry skin, nodules on the palms, calves and feet, hyperpigmentation of both calves, and contracting finger joints. A skin biopsy was obtained from the patient's right knee demonstrating thickening of the dermis and proliferating fibroblasts compatible with NSF (Fig. 1). Immunohistochemistry was positive for $\mathrm{CD} 34$ and $\mathrm{CD}$ 68. This patient had also suffered from cardiac disease (atrioventricular block) and eventually died of sudden cardiac death in 2005.

In total, seven patients died, three of cardiac death, two of natural causes, one died because the patient refused to have dialysis and one died in an accident (a fall from a cherry tree). 
Table 2 Relevant laboratory values in 27 patients post-digital subtraction angiography with gadolinium-based contrast agents

\begin{tabular}{|c|c|c|c|c|c|c|c|c|}
\hline No./sex & $\begin{array}{l}\text { sCr Level } \\
(\mu \mathrm{mol} / 1) \text { pre }\end{array}$ & $\begin{array}{l}\mathrm{sCr} \text { Level } \\
(\mu \mathrm{mol} / \mathrm{l}) 2 \mathrm{~h}\end{array}$ & $\begin{array}{l}\text { sCr Level } \\
(\mu \mathrm{mol} / 1) \\
1-2 \text { weeks }\end{array}$ & $\begin{array}{l}\text { sCr Level } \\
(\mu \mathrm{mol} / 1) \\
3 \text { months }\end{array}$ & $\begin{array}{l}\text { sCr Level } \\
(\mu \mathrm{mol} / 1) \\
\text { ultimate\# }\end{array}$ & $\begin{array}{l}\text { eGFR } \\
(\mathrm{ml} / \mathrm{min} / \\
\left.1.73 \mathrm{~m}^{2}\right) \text { pre }\end{array}$ & $\begin{array}{l}\text { eGFR, } \\
\left(\mathrm{ml} / \mathrm{min} / 1.73 \mathrm{~m}^{2}\right) \\
\text { post ultimate\# }\end{array}$ & $\begin{array}{l}\text { Chronic kidney } \\
\text { disease Stage* }\end{array}$ \\
\hline $1 / \mathrm{F}$ & 413 & 428 & NA & 316 & 350 & 10 & 13 & 5 \\
\hline $2 / \mathrm{M}$ & 292 & 285 & 269 & NA & 269 & 19 & 23 & 4 \\
\hline $3 / \mathrm{M}$ & 403 & 395 & 333 & 404 & 246 & 13 & 24 & 5 \\
\hline $4 / \mathrm{F}$ & 271 & 162 & 201 & NA & 201 & 16 & 23 & 4 \\
\hline $5 / \mathrm{F}$ & 162 & 170 & NA & NA & 170 & 28 & 27 & 4 \\
\hline $6 / F$ & 287 & 262 & 262 & 188 & 188 & 15 & 22 & 4 \\
\hline 7/M & 212 & 186 & 204 & 202 & 126 & 30 & 53 & 3 \\
\hline $8 / \mathrm{F}$ & 301 & 446 & NA & NA & 446 & 17 & 9 & 4 \\
\hline $9 / \mathrm{M}$ & 162 & 224 & 136 & 144 & 112 & 43 & 63 & 3 \\
\hline $10 / \mathrm{M}$ & 175 & 194 & 211 & 154 & 245 & 42 & 33 & 3 \\
\hline $11 / \mathrm{M}^{*}$ & 728 & 764 & 712 & 539 & 470 & 7 & 14 & 5 \\
\hline $12 / \mathrm{M}$ & 143 & 159 & 143 & NA & 149 & 45 & 42 & 3 \\
\hline $13 / F$ & 188 & 125 & NA & NA & 125 & 24 & 38 & 4 \\
\hline $14 / \mathrm{M}$ & 242 & 389 & 447 & 592 & 880 & 27 & 7 & 4 \\
\hline $15 / \mathrm{M}$ & 201 & 233 & 224 & 215 & 189 & 30 & 32 & 3 \\
\hline $16 / F$ & 350 & NA & NA & 470 & 78 & 14 & 74 & 5 \\
\hline $17 / \mathrm{M}$ & 408 & NA & NA & 562 & 631 & 14 & 7 & 5 \\
\hline $18 / \mathrm{M}$ & 136 & NA & NA & NA & 132 & 53 & 53 & 3 \\
\hline $19 / F$ & 266 & NA & NA & 126 & 126 & 16 & 37 & 4 \\
\hline $20 / \mathrm{M}$ & 323 & 338 & NA & NA & 329 & 17 & 17 & 4 \\
\hline $21 / F$ & NA & NA & 127 & NA & 113 & NA & 42 & NA \\
\hline $22 / \mathrm{M}$ & 229 & 230 & NA & NA & 251 & 27 & 25 & 4 \\
\hline $23 / F$ & 177 & NA & NA & NA & 177 & 27 & 27 & 4 \\
\hline $24 / \mathrm{M}$ & 177 & NA & NA & NA & 308 & 36 & 18 & 3 \\
\hline $25 / \mathrm{M}$ & 144 & 197 & 129 & 122 & 109 & 44 & 59 & 3 \\
\hline $26 / \mathrm{M}$ & 317 & NA & 266 & NA & 140 & 18 & 45 & 4 \\
\hline $27 / \mathrm{M}$ & 134 & NA & NA & NA & 111 & 49 & 62 & 3 \\
\hline MEAN & 263.1 & 288.2 & 261.7 & 310.3 & 247.1 & 26.2 & 32.9 & 3.8 \\
\hline MEDIAN & 235.5 & 231.5 & 217.5 & 215 & 188 & 25.5 & 27 & 4 \\
\hline SD & 128.7 & 154.7 & 156.6 & 179.5 & 181.8 & 13.1 & 18.5 & 0.7 \\
\hline MIN & 134 & 125 & 127 & 122 & 78 & 7 & 7 & 3 \\
\hline MAX & 728 & 764 & 712 & 592 & 880 & 53 & 63 & 5 \\
\hline
\end{tabular}

*This patient demonstrated NSF-typical skin changes that were biopsy-proven

Based on National Kidney Foundation Kidney Disease Outcomes Quality Initiative: eGFR values for stage 1, >90 ml $/ \mathrm{min}_{2} / 1.73 \mathrm{~m}$; $\mathrm{stage} 2$, $60-90 \mathrm{ml} / \mathrm{min} / 1.73 \mathrm{~m}^{2}$; stage 3, 30-59 ml/min $/ 1.73 \mathrm{~m}^{2}$; stage $4,15-29 \mathrm{ml} / \mathrm{min} / 1.73 \mathrm{~m}^{2}$; stage $5,<15 \mathrm{ml} / \mathrm{min} / 1.73 \mathrm{~m}{ }^{2}$

NA, Not available; M, male; F, female; SD, standard deviation; eGFR, estimated glomerular filtration rate

\#Mean follow-up period was 28 months, range $1-84$ months

\section{Discussion}

In the present study we reviewed clinical and laboratory data of 27 patients with renal insufficiency who underwent conventional angiography using intra-arterial gadoliniumbased contrast agents (GDBCA) as alternate $\mathrm{x}$-ray contrast media and assessed the occurrence of nephrogenic systemic fibrosis. Additional MRI studies with GDBCA were performed in 15 patients. Despite this rather high total exposure to gadolinium-based contrast agents, only one patient demonstrated NSF-typical skin changes that were biopsy-proven. We cannot exclude further cases, but these at least were not spontaneously reported or registered within the clinical records.

It is well established that the use of iodinated contrast material may cause contrast-induced nephropathy (CIN), 
Table 3 Data compilation of a 20-year old patient with glomerulonephritis and tubulointerstitial nephritis. The patient was on dialysis from October 1998 to August 2000. He received a renal transplant in August 2000, and dialysis was stopped. The patient underwent multiple MRA studies with GDBCA post-renal transplantation receiving both linear and macrocyclic gadolinium-based contrast agents. The patient was examined on 17 April 2001 for conventional angiography with $15 \mathrm{ml}$ of intra-arterial gadodiamide. $\mathrm{He}$ had to go back on dialysis in May 2001. He observed skin changes and developed joint pain in September 2001. At the time, the patient had received a total of $215 \mathrm{ml}$ of GDBCA, $135 \mathrm{ml}$ of linear and $80 \mathrm{ml}$ of macrocyclic contrast agents. A skin biopsy taken in late December 2001 was positive for NSF in retrospect

\begin{tabular}{|c|c|c|c|c|c|c|}
\hline Date & Study & Type of GDBCA & Structure of GDBCA & Amount of GDBCA & Creatinine $(\mu \mathrm{mol} / \mathrm{l})$ & $\mathrm{eGFR}\left(\mathrm{ml} / \mathrm{min} / 1.73 \mathrm{~m}^{2}\right)$ \\
\hline 11.08 .00 & MRI renal & ProHance & macrocyclic & $30 \mathrm{ml}$ & 598 & 10 \\
\hline 22.08 .00 & MRI renal & Omniscan & linear & $30 \mathrm{ml}$ & 607 & 10 \\
\hline 04.09 .00 & MRI thigh & ProHance & macrocyclic & $30 \mathrm{ml}$ & NA & NA \\
\hline 21.09 .00 & MRI renal & Omniscan & linear & $30 \mathrm{ml}$ & NA & NA \\
\hline 23.11 .00 & MRI renal & Omniscan & linear & $30 \mathrm{ml}$ & 617 & 10 \\
\hline 10.04 .01 & MRI renal & Omniscan & linear & $30 \mathrm{ml}$ & NA & NA \\
\hline 17.04 .01 & Angio renal transplant & Omniscan & linear & $15 \mathrm{ml}$ & 728 & 7 \\
\hline 24.12.01 & MRI renal & ProHance & macrocyclic & $20 \mathrm{ml}$ & 725 & 8 \\
\hline 26.03 .03 & MRI knee & Magnevist & linear & $10 \mathrm{ml}$ & 660 & 9 \\
\hline
\end{tabular}

GDBCA, gadolinium-based contrast agent; NA, not available; eGFR, estimated glomerular filtration rate

especially in patients with underlying renal insufficiency [1]. Because GDBCA seemed to have a reduced nephrotoxicity compared with iodinated agents, physicians were encouraged to switch over to GDBCA [11]. However, it was a previous misconception that the use of GDBCA was safe in patients with underlying renal insufficiency [2]. The Contrast Media Safety Committee (CMSC) of the European Society of Urogenital Radiology (ESUR) did not approve GDBCA for radiographic examinations [3]. It was stated that GDBCA should not be used for radiographic examinations in patients with renal impairment and that these contrast agents are more nephrotoxic than iodinated contrast in equivalent radiographic attenuating doses.

In a previous study, GDBCAs were used to perform digital subtraction angiography and percutaneous treatment of renal artery stenosis in such patients [4]. Although contrast-induced nephropathy potentially occurred in no more than $3.2 \%$ of patients in this study, recent findings indicate that these patients may be at increased risk of NSF.

In our study, all patients had an alteration of renal function. As reported by Sadowski et al., a combination of factors, including altered renal function, chronic inflammation and exposure to gadolinium-based contrast agents, may all play a role in the development of NSF [12]. In their study, data of 13 patients with biopsy-confirmed NSF were reviewed, and associated risk factors were assessed. All patients had been exposed to GDBCA within a time frame of 6 months. All patients had renal insufficiency and had been hospitalised for a proinflammatory event such as surgery, infection or a vascular event. Compared with a control group, these patients had significantly decreased eGFR, more proinflammatory events and more MRI studies with GDBCA. In our study, conventional arterial angiography was performed, which represents a proinflammatory element that basically increases the risk of NSF. Furthermore,
GDBCA was administered intra-arterially for conventional angiography. This implies higher renal first-pass toxicity compared with IV administration, especially if selective renal artery angiography was performed.

Nephrogenic systemic fibrosis occurring in patients with kidney impairment was lately reported to present with characteristic skin manifestation and joint disease $[5,13$, 14]. Previous literature reports describe coherence between the use of gadolinium-based contrast agents and NSF [8$10,15-17]$. In our study, one patient demonstrated skin lesions characteristic of NSF, which was biopsy-proven. There was a hiatus of approximately 5 months between his renal transplant angiography with GDBCA and the onset of symptoms, which seems rather long according to the European Society of Urogenital Radiology (ESUR) guidelines, defining 8 weeks as the standard critical period [18]. As mentioned earlier, this patient had received as much as $135 \mathrm{ml}$ of linear GDBCA before the onset of symptoms. It remains uncertain if NSF was caused by the single-dose of GDBCA administered during catheter angiography, while the renal impairment was almost complete or the ones given earlier for several MRI studies of the patient's kidney and thigh or the total amount of gadolinium during the patient's life-time. However, the cumulative dose of GDBCA seems to be more likely responsible for the onset of NSF than a single dose of $15 \mathrm{ml}$ administered during angiography [19].

We propose that there may be a possibility that repeated GDBCA administration, as in our case, increased the risk of NSF occurring in terms of an additive effect in GDBCA deposition $[10,19,20]$. Furthermore, Abraham et al. investigated retained gadolinium-containing deposits in skin biopsies from 20 patients with gadolinium-related NSF [21]. Of interest, gadolinium concentration increased over time with multiple sequential biopsies in more than 


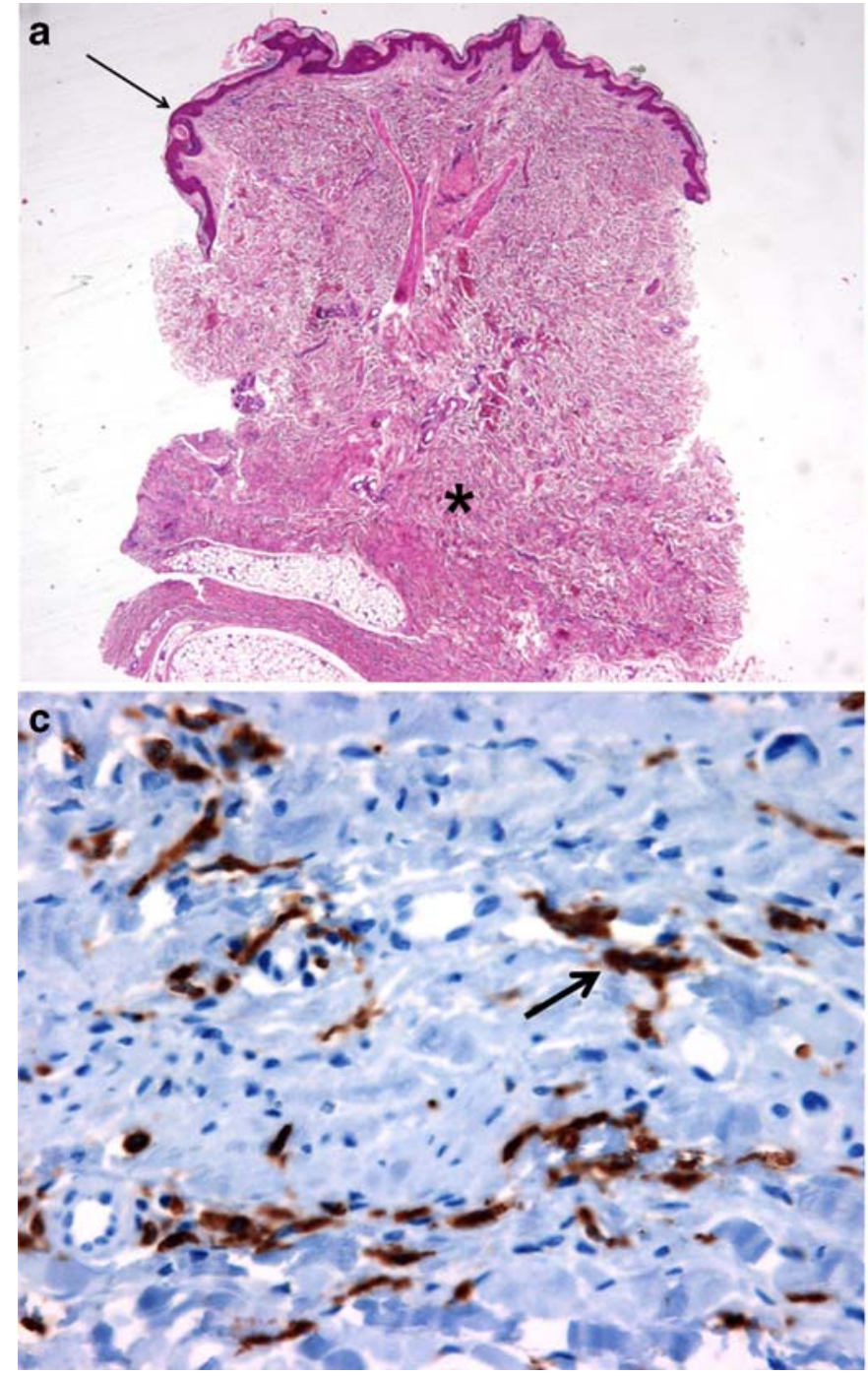

Fig. 1 Skin biopsy specimen obtained from patient's right knee. a, Biopsy (haematoxylin and eosin) with low magnification demonstrates thickening of the dermis (arrow) with haphazardly arranged collagen bundles separated by large clefts in the papillary and deep dermis. Spindled fibroblasts extend into subcutaneous septae (asterisk). b High magnification with an increased number of

$50 \%$ of patients for 23 months; after that a rather slow decrease was observed reflecting the difficulty in eliminating the gadolinium once it is deposited in the tissues. The authors suggested that gadolinium may be mobilised over time from bone stores, explaining the variably delayed onset of NSF.

The liberation of toxic and insoluble gadolinium after dechelation following transmetallation of the gadolinium chelate is the proposed trigger for NSF [8]. Free gadolinium ions are released from the chelate in exchange for endogenous metals, protons and subsequently form insoluble phosphates or hydroxides in the peripheral human tissues. The precipitating gadolinium salts are engulfed by circulating macrophages that attract fibrocytes. Because of
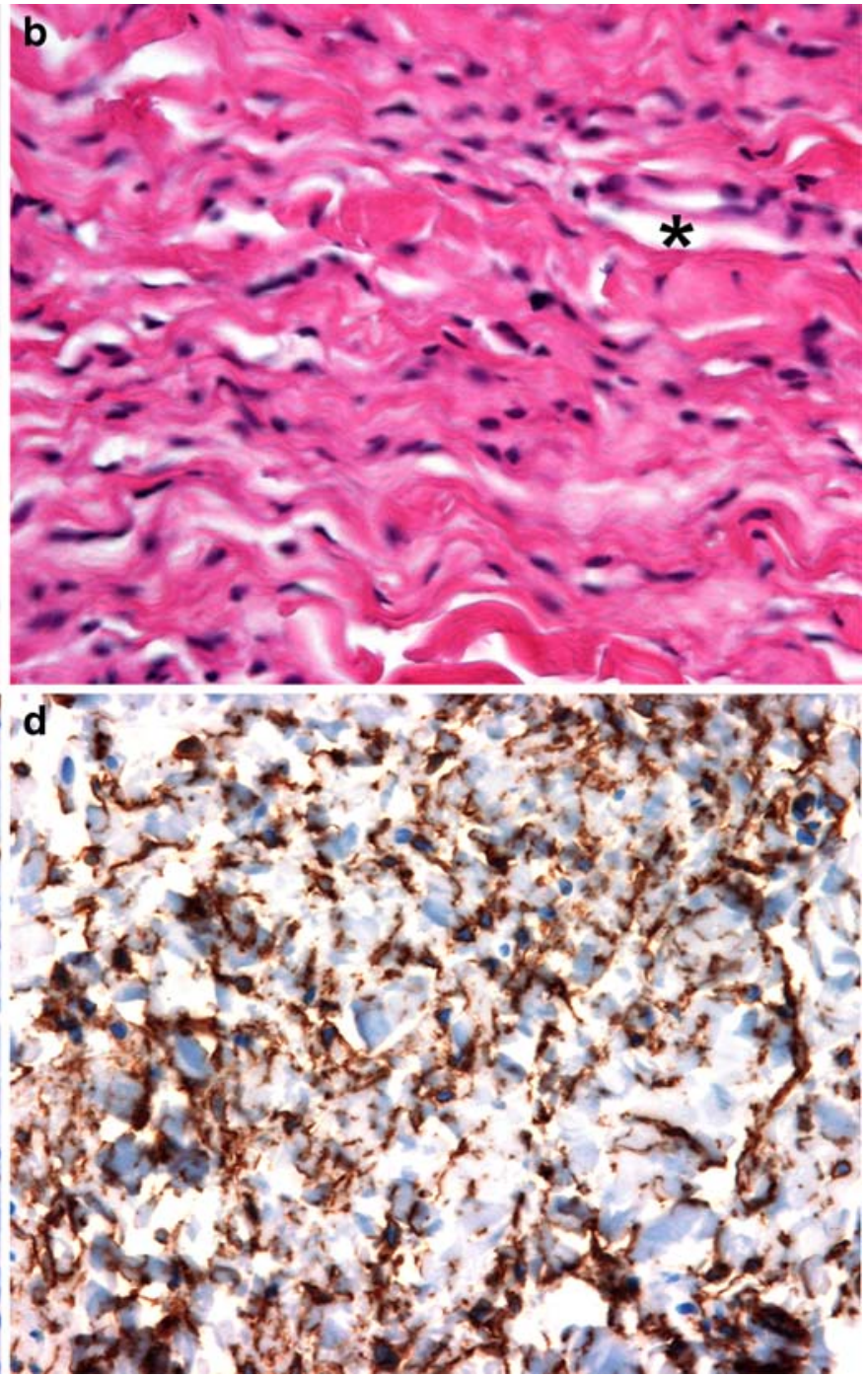

spindled fibroblasts. Collagen bundles are separated by large clefts (asterisk). c High magnification (immunohistochemical stain with CD 68) demonstrates positive stellate fibroblast cells (arrow). d High magnification (immunohistochemical stain with CD 34) demonstrates fibroblast reactivity

the relatively high capacity of solubilisation within the lysosomes of the macrophages, these cells are probably intoxicated, leading to a vicious circle. Because of a markedly prolonged clearance and circulation time of GDBCA in patients with end-stage renal disease, these patients are at increased risk of NSF development. Mainly two types of GDBCA chelates, linear open-chain and macrocyclic ones, are commercialised. They differ in their thermodynamic stability ratios expressed at different $\mathrm{pH}$ values and velocity of decomplexation when under competition from other protons or electrolytes. Linear chelates, such as gadodiamide (Omniscan), which was mainly used for angiography in our study because of its rather low osmolality of $780 \mathrm{mOsm} / \mathrm{kg} \mathrm{H} \mathrm{H}_{2} \mathrm{O}$ at $37^{\circ} \mathrm{C}$, seem to be at 
higher risk of decomplexation than macrocylic chelates such as gadoteridol (ProHance). A summary of the medical literature reports more than $90 \%$ of NSF cases associated with GDBCA administration related to gadodiamide (Omniscan) [22]. Thomsen et al. report the prevalence of NSF to be significantly higher post-gadodiamide than any other gadolinium-based agent $(3-7 \%$ versus $0-1 \%$ per injection) in patients with reduced renal function [17]. After exposure to two gadodiamide injections, they found prevalence as high as $36 \%$ in patients with chronic kidney disease stage 5 . In the current literature, no reports of NSF after the administration of most stable agents have been published, implying that there may be a difference in triggering NSF between various agents.

As the relationship of NSF with the administration of GDBCA was unknown at the time, absurdly, additional MRI studies with gadolinium-based contrast agents were performed working up our patient's symptoms and following up the renal transplant. Despite the nebulosity of NSF at the time, in our positive case a skin biopsy was obtained, and histopathology was reported to demonstrate dermal fibrosis, thickening of the dermis and proliferating fibroblasts. With today's knowledge, these findings are compatible with NSF. Immunohistochemistry was not performed at the time, but we were able to obtain the original tissue specimen and produced additional stains using immunohistochemistry for CD 34 and CD 68, which were characteristically positive for NSF.

There are several limitations of this study. First, the study is retrospective and therefore not controlled for selection bias, detection of events and data collection. Second, although 27 patients were studied, adverse events that occur at a low rate or that were of rather weak intensity could have been missed or were underreported. Moreover, different types of GDBCA were used in our study, further decreasing the potential sensitivity of the study for adverse events and correlation of GDBCA with outbreaks of NSF. Third, because of the retrospective design of this study, signs for NSF could have been missed in individual patients because data were missing and because NSF had not been recognised during the entire study period included.

In conclusion, exposition with gadolinium-based contrast agents during conventional angiography may play a role in the development of NSF in patients with renal insufficiency. Alternative contrast agents such as $\mathrm{CO}_{2}$ angiography or rather the use of low doses of iodinated contrast agents should be considered in these patients.

\section{References}

1. Spinosa DJ, Angle JF, Hagspiel KD, Kern JA, Hartwell GD, Matsumoto AH (2000) Lower extremity arteriography with use of iodinated contrast material or gadodiamide to supplement $\mathrm{CO}_{2}$ angiography in patients with renal insufficiency. J Vasc Interv Radiol 11:35-43

2. Spinosa DJ, Matsumoto AH, Angle JF, Hagspiel KD, McGraw JK, Ayers C (1999) Renal insufficiency: usefulness of gadodiamide-enhanced renal angiography to supplement $\mathrm{CO}_{2}$-enhanced renal angiography for diagnosis and percutaneous treatment. Radiology 210:663-672

3. Thomsen HS, Almen T, Morcos SK (2002) Gadolinium-containing contrast media for radiographic examinations: a position paper. Eur Radiol 12:2600-2605

4. Spinosa DJ, Matsumoto AH, Angle JF, Hagspiel KD, Cage D, Bissonette EA et al (2001) Safety of CO(2)- and gadodiamide-enhanced angiography for the evaluation and percutaneous treatment of renal artery stenosis in patients with chronic renal insufficiency. AJR Am J Roentgenol 176:1305-1311
5. Cowper SE (2005) Nephrogenic systemic fibrosis: the nosological and conceptual evolution of nephrogenic fibrosing dermopathy. Am J Kidney Dis 46:763-765

6. Galan A, Cowper SE, Bucala R (2006) Nephrogenic systemic fibrosis (nephrogenic fibrosing dermopathy). Curr Opin Rheumatol 18:614-617

7. Gibson SE, Farver CF, Prayson RA (2006) Multiorgan involvement in nephrogenic fibrosing dermopathy: an autopsy case and review of the literature. Arch Pathol Lab Med 130:209-212

8. Grobner T (2006) Gadolinium-a specific trigger for the development of nephrogenic fibrosing dermopathy and nephrogenic systemic fibrosis? Nephrol Dial Transplant 21:1104-1108

9. High WA, Ayers RA, Chandler J, Zito $\mathrm{G}$, Cowper SE (2007) Gadolinium is detectable within the tissue of patients with nephrogenic systemic fibrosis. J Am Acad Dermatol 56:21-26

10. Thomsen HS, Marckmann P, Logager VB (2007) Nephrogenic systemic fibrosis (NSF): a late adverse reaction to some of the gadolinium based contrast agents. Cancer Imaging 7:130-137

11. Sam AD 2nd, Morasch MD, Collins J, Song G, Chen R, Pereles FS (2003) Safety of gadolinium contrast angiography in patients with chronic renal insufficiency. J Vasc Surg 38:313-318
12. Sadowski EA, Bennett LK, Chan MR, Wentland AL, Garrett AL, Garrett RW et al (2007) Nephrogenic systemic fibrosis: risk factors and incidence estimation. Radiology 243:148-157

13. Hope TA, Herfkens RJ, Denianke KS, Leboit PE, Hung YY, Weil E (2009) Nephrogenic systemic fibrosis in patients with chronic kidney disease who received gadopentetate dimeglumine. Invest Radiol. doi:10.1097/RLI.0b013e31819343ba

14. Janus N, Launay-Vacher V, Karie S, Clement O, Ledneva E, Frances $\mathrm{C}$ et al (2009) Prevalence of nephrogenic systemic fibrosis in renal insufficiency patients: results of the FINEST study. Eur J Radiol. doi:10.1016/j. ejrad.2008.11.021

15. Perazella MA (2009) Current status of gadolinium toxicity in patients with kidney disease. Clin J Am Soc Nephrol 4:461-469

16. Perez-Rodriguez J, Lai S, Ehst BD, Fine DM, Bluemke DA (2009) Nephrogenic systemic fibrosis: incidence, associations, and effect of risk factor assessment-report of 33 cases. Radiology 250:371-377 
17. Thomsen HS, Marckmann P (2008) Extracellular Gd-CA: differences in prevalence of NSF. Eur J Radiol 66:180-183

18. Thomsen HS (2007) ESUR guideline: gadolinium-based contrast media and nephrogenic systemic fibrosis. Eur Radiol 17:2692-2696
19. Collidge TA, Thomson PC, Mark PB, Traynor JP, Jardine AG, Morris ST et al (2007) Gadolinium-enhanced MR imaging and nephrogenic systemic fibrosis: retrospective study of a renal replacement therapy cohort. Radiology 245:168-175

20. Thakral C, Alhariri J, Abraham JL (2007) Long-term retention of gadolinium in tissues from nephrogenic systemic fibrosis patient after multiple gadolinium-enhanced MRI scans: case report and implications. Contrast Media Mol Imaging 2:199-205
21. Abraham JL, Thakral C, Skov L, Rossen K, Marckmann P (2008) Dermal inorganic gadolinium concentrations: evidence for in vivo transmetallation and long-term persistence in nephrogenic systemic fibrosis. $\mathrm{Br} \mathrm{J}$ Dermatol 158:273-280

22. Broome DR (2008) Nephrogenic systemic fibrosis associated with gadolinium based contrast agents: a summary of the medical literature reporting. Eur J Radiol 66:230-234 